\title{
On the asymptotic equivalence between stochastic and deterministic energy sources in energy harvesting sensing systems
}

\author{
Jingxian $\mathrm{Wu}^{*}$ and Jing Yang
}

\begin{abstract}
In this paper, we seek answer to the question: can a wireless sensing system with energy harvesting power supplies perform as well as the one with conventional power supplies? Conventional sensing systems with deterministic energy sources usually employ uniform sampling. However, due to the stochastic nature of the energy harvested from the ambient environment, uniform sampling is usually infeasible for energy harvesting sensing systems. We thus propose a simple best-effort sensing scheme, which defines a set of equally spaced candidate sensing instants. At a given candidate sensing instant, the sensor will perform sensing if there is sufficient energy available, and it will remain silent otherwise. It is analytically shown that the percentage of silent candidate sensing instants goes to zero as time increases, if and only if the average energy harvesting rate is no less than the average energy consumption rate. Therefore, the difference between the best-effort sensing policy and the uniform sensing policy diminishes as time evolves. The theoretical results are then used to guide the design of a practical sensing system that monitors a time-varying event. Both analysis and simulations show that the energy harvesting system with the best-effort sensing scheme can asymptotically achieve the same mean squared error (MSE) performance as the one with uniform sensing and deterministic energy sources. Therefore, we provide a positive answer to the question by establishing the asymptotic equivalence between stochastic and deterministic energy sources, from both theoretical and practical aspects.
\end{abstract}

Keywords: Energy harvesting; Stochastic energy sources; Asymptotic equivalence; MSE

\section{Introduction}

Wireless sensing systems, such as structure health monitoring, biomedical sensing, and environment monitoring, are usually expected to operate uninterruptedly and autonomously over years or decades under extremely stringent energy constraints. Such design objectives necessitate the development of systems powered by energy harvesting devices, such as piezoelectric, thermal, or photovoltaic devices, which can collect energy from the ambient environment. Due to the randomness of the energy harvesting process, the amount of harvested energy can be modeled as a stochastic process. The stochastic nature of the energy sources is fundamentally different from the deterministic energy sources employed

*Correspondence:wuj@uark.edu

Department of Electrical Engineering, University of Arkansas, Fayetteville, AR 72701, USA by conventional systems, and it imposes formidable challenges on the development of energy harvesting sensing systems.

There have been growing interests in the development of energy harvesting communications and sensing systems. Many of the works are developed to optimize communication-related metrics, such as transmission delay or throughput. In [1], the transmission delay of a point-to-point system is minimized by considering causality constraints on both information and energy, i.e., information cannot be delivered before its arrival and energy cannot be consumed before it is harvested. The formulation in [1] is extended to broadcast channels in $[2,3]$ and to a multiple access channel in [4]. All of the above works use an off-line deterministic scheduling method, which identifies the optimum transmission scheduling based on full knowledge of current and future energy arrivals. The off-line scheduling methods treat future energy arrival

\section{Springer}

(c) 2015 Wu and Yang; licensee Springer. This is an Open Access article distributed under the terms of the Creative Commons Attribution License (http://creativecommons.org/licenses/by/4.0), which permits unrestricted use, distribution, and reproduction in any medium, provided the original work is properly credited. 
as a deterministic process even though the actual energy harvesting process is stochastic.

Online scheduling methods address this problem by using only the statistics of energy arrivals. The off-line and online scheduling for systems with fading channels are discussed in [5], where the online scheduling problem is formulated as a stochastic dynamic programming problem with high complexity. Low complexity sub-optimum online algorithms are presented in [5] for fading channel and in [6] for interference channel. In both works, the performance of all online scheduling policies is strictly worse than that of the off-line scheduling.

Another branch of work focuses on sensing and signal processing-related performance metrics, such as estimation mean squared error (MSE), probability of false alarm and/or missed detection, and detection delay. In [7], the estimation MSE of a sparse signal is minimized by using compressive sensing and a random transmission scheme, where each energy harvesting sensor node transmits with a certain probability under the energy causality constraint. The sensing energy is assumed to be negligible in [7]. Optimum energy allocation schemes are discussed in [8] for the 'quickest detection' of the changing point of an event monitored by energy harvesting sensor networks. The results in [8] do not consider distortions such as sensing noise. Generally speaking, the energy management policies for the optimization of performance metrics related to sensing and inference have been less investigated.

All of the above works show that the performance of energy harvesting systems is in general inferior to systems with conventional deterministic energy sources, even if optimal sensing policies are developed based on the stochastic properties of the energy sources. The performance loss of the energy harvesting systems is mainly due to the stochastic nature of the energy source, such that energy is not always available when needed for sensing or transmission.

Naturally, we would ask the question: can an energy harvesting sensing system with stochastic energy sources perform as well as a conventional sensing system powered by deterministic energy sources? We seek the answer to this question from two aspects: from the theoretical aspect in terms of the stochastic properties of the available energy at a given time, and from the practical aspect in terms of the MSE performance of a sensing system. For a conventional sensing system with deterministic energy sources, uniform sampling is usually employed by the sensors and it is optimum over the duration in which the signal is wide sense stationary (WSS) $[9,10]$. However, due to the randomness in the energy arrival process, uniform sampling might be infeasible in energy harvesting systems given that there might not be sufficient energy to perform sensing operations at certain time periods.
We thus propose a simple best-effort sensing policy that attempts to achieve uniform sampling with its best efforts [11-13]. The best-effort policy defines a set of equally spaced candidate sensing instants. At a given candidate sensing instant, the sensor will perform sensing only if there is sufficient energy to do so, and it will remain silent otherwise.

By analyzing the stochastic properties of the energy sources, we will show that the percentage of silent candidate sensing instants goes to zero as time goes to infinity, if and only if the average energy collection rate is no less than the average energy consumption rate. This means that the difference between the best-effort sensing policy and the uniform sensing policy diminishes as time evolves. The theoretical results indicate that the sensing behavior of systems with stochastic energy sources has the potential to approach that of systems with deterministic energy sources. Guided by the theoretical results, we then develop optimum sensing and detection schemes for a practical energy harvesting sensing system used to monitor a time-varying event. It will be shown through both theoretical analysis and simulations that the MSE performance of the energy harvesting sensing system asymptotically approaches that of a conventional sensing system with uniform sensing as time goes to infinity. Simulation results show that the performance of the two systems converges with as few as 400 candidate sensing instants. Therefore, we demonstrate from both theoretical and practical aspects that there is an asymptotic equivalence between stochastic and deterministic energy sources.

The remainder of this paper is organized as follows. The problem formulation is given in Section 2 . Section 3 demonstrates the asymptotic equivalence between stochastic and deterministic energy sources by showing that the percentage of silent sensing instants goes to zero as time goes to infinity. The theoretical results are then applied in Section 4 to guide the development of a practical energy harvesting sensing system that can asymptotically achieve the same MSE performance as a conventional sensing system. Numerical and simulation results are given in Section 5, and Section 6 concludes the paper.

\section{Problem formulation}

Consider a sensor used to monitor a time-varying event. The sensor is powered by an energy harvesting device, which harvests energy from the ambient environment. Due to the random nature of the energy harvesting process, the amount of harvested energy can be modeled as a random process. The harvested energy is used by the sensor for sensing operations.

The time-varying event being monitored is modeled as a WSS random process $s(t)$, where $t$ is the time variable. It 
is assumed that $s(t)$ is zero mean with a power-law autocovariance function

$$
\mathbb{E}\left[s\left(t_{1}\right) s\left(t_{2}\right)\right]=\rho^{\left|t_{1}-t_{2}\right|}
$$

where $\mathbb{E}$ is the mathematical expectation operator, and $0 \leq \rho \leq 1$ is the power-law coefficient. The powerlaw covariance is an alternative representation of the Ornstein-Uhlenbeck covariance kernel [14].

The sensor attempts to reconstruct the continuoustime time-varying random event by using noise-distorted discrete-time observations of the random process. A sensing policy is defined as a sequence of time instants $\left\{t_{n}\right\}_{n}$, where $t_{n}$ is the time instant at which the sensor collects a sample of the random process. The sample collected by the sensor at time $t_{n}$ is

$$
y\left(t_{n}\right)=\sqrt{E_{s}} s\left(t_{n}\right)+z\left(t_{n}\right)
$$

where $E_{s}$ is the energy allocated for one sensing sample, and $z\left(t_{n}\right)$ is the sensing and/or channel noise with a zeromean and the auto-covariance function $\mathbb{E}\left[z\left(t_{1}\right) z\left(t_{2}\right)\right]=$ $\sigma_{z}^{2} \delta\left(t_{1}-t_{2}\right)$. It should be noted that the noise component is not necessarily Gaussian distributed.

The sensing system attempts to reconstruct the timevarying random field, $s(t)$, by using the sequence of the discrete-time samples, $\left\{y\left(t_{n}\right)\right\}_{n}$. If the sensor is powered by a conventional power supply, then uniform sampling with $t_{n}=n T_{s}$ is employed in $[9,10]$ to achieve the optimum sensing performance due to the homogeneous nature of the random field. However, due to the inherent randomness of the energy harvesting process, uniform sampling might be infeasible given that there might not be sufficient energy to perform sensing operations at certain time periods. The performance of uniform sampling with conventional power supply can then serve as a lower bound for systems with energy harvesting devices.

\section{Asymptotic equivalence between stochastic and deterministic energy sources}

In this section, we first present a best-effort sensing policy for systems with stochastic energy sources. We then study the asymptotic behaviors of the best-effort sensing policy as time goes to infinity. The analytical results demonstrate the asymptotic equivalence between stochastic energy sources and deterministic energy sources.

Due to the random nature of the energy harvesting process, the harvested energy can be modeled as a random process. We model the stochastic energy sources by using the following two assumptions:

Assumption 1. If we divide the time axis into arbitrary small intervals with length $\Delta>0$, then the energy collected in each interval can be modeled as independently and identically distributed (i.i.d.) random variables, $E_{\Delta}=$ $P \Delta$, where $P$ is the average energy harvesting rate.
Assumption 2. $\sum_{n=1}^{\infty} \operatorname{Pr}\left(E_{\Delta}>n \epsilon\right)<\infty$ for any $\epsilon>0$ and $\Delta>0$.

Assumption 1 indicates that the energy collected at different time intervals are independent, with the average amount of harvested energy proportional to the time duration, and Assumption 2 is used to ensure that the model behaves normally. Such a model is general enough to incorporate many other existing stochastic energy models as special cases. Below are a few examples.

1. Poisson energy source [5]. The energy arrives in the form of energy packets with unit energy in each energy packet. The number of energy arrivals in a unit time is a Poisson random variable with mean $\lambda$. Thus, the average energy collected in an interval $\Delta$ is $E_{\Delta}=\lambda \Delta$, and the amount of energy collected in different intervals is i.i.d.

2. Bernoulli energy source [8]. In each interval $\Delta$, the energy arrival can be modeled as an i.i.d. Bernoulli random variable with parameter $P(E=1)=p_{\Delta}$. The average energy collected in an interval $\Delta$ is thus $E_{\Delta}=p_{\Delta}$.

3. Gaussian energy source. The energy source is modeled as $E=|V(t)|^{2}$, where $V(t)$ is a zero-mean symmetric complex Gaussian random process with covariance function $\mathbb{E}\left[V(t+\tau) V^{*}(t)\right]=\sigma_{v}^{2} \delta(\tau)$. The average energy collected in an interval $\Delta$ is $E_{\Delta}=\sigma_{v}^{2} \Delta$.

The Poisson and Bernoulli energy sources model the energy arrivals with discrete random variables, and they are widely used in the literature. The Gaussian energy source uses continuous random variables to model the energy arrival, and it can be used to model the energy collected from random events that have similar properties as white Gaussian noise. All three models fall in the general stochastic energy model. Other than the two assumptions, the analysis in the rest of this paper does not assume any specific distributions of the energy sources.

The harvested energy is stored in an energy storage device, such as rechargeable batteries or super capacitors. The energy storage device can be modeled as an energy queue, with the input being the random energy arrival from the energy harvesting process, and the output is the energy consumption. Denote the amount of energy available in the energy storage device at time $t$ as $Q(t) \geq 0$. Since the harvested energy is usually very small compared to the capacity of the energy storage device, it is assumed that the energy queue has unlimited capacity. The energy consumption must follow the energy causality constraint, that is, at any time instant, the total amount of harvested energy must be no less than the total amount of consumed energy. 
The amount of energy available in the energy queue, $Q(t)$, is a random process due to the randomness in both energy collections and energy consumptions. During some time instants, there might not be enough energy in the energy queue for the sensing operations, that is, $Q(t)<E_{s}$. Therefore, the uniform sensing policy for systems with a deterministic power supply is not applicable to energy harvesting systems. We propose a best-effort sensing policy defined as follows.

Definition 1 (Best-effort sensing policy). Define a set of candidate sensing instants as $\mathcal{K}=\left\{k_{n} \mid k_{n}=n T_{s}, n=\right.$ $1,2, \cdots\}$. A sensor performs one sensing operation with energy $E_{s}$ at time $t$ if and only if: 1) $t \in \mathcal{K}$, and 2) $Q(t) \geq E_{s}$.

In the best-effort sensing policy, the sensor attempts to mimic the uniform sensing policy with its best efforts. It tries to perform sensing operations at uniform sensing intervals whenever allowed by the energy constraint. However, it will keep silent at a candidate sensing instant $n T_{s}$ if $Q\left(n T_{s}\right)<E_{s}$. Denote the information collected at each candidate sensing instant as a sensing symbol, which could be either a silent symbol when $Q\left(n T_{s}\right)<E_{s}$ or an active symbol when $Q\left(n T_{s}\right) \geq E_{s}$. With such a sensing mechanism and the stochastic energy source, there might be $K$ silent symbols in the first $N \geq K$ sensing instants $T_{s}, 2 T_{s}, \cdots, N T_{s}$. The number of silent symbols is a random variable. The existence of silent symbols might degrade the sensing performance.

Next, we show the first main result of this paper, which states that if certain conditions are satisfied, then there is a diminishing number of silent symbols compared to active symbols as time becomes large enough.

Theorem 1. Consider an energy harvesting sensing system powered by energy sources satisfying Assumptions 1 and 2. The system employs the best-effort sensing policy described in Definition 1. Define $K=\sum_{k=1}^{N} \mathbf{1}_{Q\left(k T_{s}\right)<E_{s}}$ as the total number of silent symbols in the first $N$ symbol periods, where the indicator function $\mathbf{1}_{\mathcal{E}}=1$ if the event $\mathcal{E}$ is true and 0 otherwise.

$$
\begin{aligned}
& \text { If } P \geq \frac{E_{s}}{T_{s}}, \text { then } \\
& \lim _{N \rightarrow \infty} \frac{K}{N}=0, \quad \text { a.s. }
\end{aligned}
$$

Specifically, if $P>\frac{E_{s}}{T_{s}}$, then for almost every energy harvesting sample path, there exists $\bar{K}<\infty$ such that $K \leq$ $\bar{K}<\infty$ as $N \rightarrow \infty$.

Conversely, if $P<\frac{E_{s}}{T_{s}}$, then

$$
\lim _{N \rightarrow \infty} \frac{K}{N} \geq 1-\frac{P T_{s}}{E_{s}}, \text { a.s. }
$$

Proof. Define $E_{c}=P T_{s}$ as the average energy harvested in the duration of $T_{s}$. Divide the time axis into frames, each of duration $L T_{s}$. The $m$-th frame thus has $L$ candidate sensing instants, and $k_{m} \leq L$ of them are assumed to be silent. Assume the total amount of energy collected in the $m$-th frame is $E_{m}$, which is random. The amount of energy consumed in the $l$-th frame can be calculated as $\left(L-k_{m}\right) E_{s}$. Denote $D_{m}=E_{m}-\left(L-k_{m}\right) E_{s}$ as the difference between the energy harvested and consumed in the $m$-th frame. It should be noted that $D_{m}$ could be either positive or negative. The total amount of energy available in the energy queue at the end of the $m$-th frame is $Q\left(m L T_{s}\right)=$ $\sum_{m=1}^{M} D_{m}$. It should be noted that $\sum_{m=1}^{M} D_{m} \geq 0$ due to the energy causality constraint.

With the best-effort sensing policy, the number of silent symbols in the $M$-th frame must satisfy

$$
k_{M} \leq \max \left\{0, L-\frac{1}{E_{s}} \sum_{m=1}^{M-1} D_{m}\right\},
$$

because the energy available at the end of the $(M-1)$-th frame can be used for the sensing of up to $\frac{1}{E_{s}} \sum_{m=1}^{M-1} D_{m}$ symbols in the $M$-th frame.

1) Case 1: $P<\frac{E_{s}}{T_{s}}$. With the energy causality constraint, we have $\sum_{m=1}^{M} D_{m} \geq 0$, or

$$
\sum_{m=1}^{M} E_{m}-M L E_{s}+E_{s} \sum_{m=1}^{M} k_{m} \geq 0
$$

Divide both sides of (6) by $M L E_{s}$, and let $M \rightarrow \infty$,

$$
\lim _{M \rightarrow \infty} \frac{\sum_{m=1}^{M} k_{m}}{M L} \geq 1-\frac{1}{E_{s}} \lim _{M \rightarrow \infty} \frac{\sum_{m=1}^{M} E_{m}}{M L}
$$

Based on the strong law of large numbers,

$$
\lim _{M \rightarrow \infty} \frac{\sum_{m=1}^{M} E_{m}}{M L}=E_{c} \text {, a.s. }
$$

In addition, let $N=L M$, then $K=\sum_{m=1}^{M} k_{m}$. Thus, (4) can be obtained from (7) and (8).

2) Case 2: $P=\frac{E_{s}}{T_{s}}$. Index the frames with at least one silent symbol as $M_{1}, M_{2}, \cdots, M_{i}, \cdots$, i.e.,

$$
0<k_{M_{i}} \leq L-\frac{\sum_{m=1}^{M_{i}-1} D_{m}}{E_{s}} .
$$

If $M_{i}$ is upper bounded, that is, there exists $\bar{M}$ such that $k_{m}=0$ for all $m>\bar{M}$, then $K$ is finite and (3) is true. On the other hand, if $M_{i}$ is unbounded, then $\lim _{i \rightarrow \infty} M_{i}=\infty$. We have

$$
\sum_{m=1}^{M_{i}} D_{m}=\sum_{m=1}^{M_{i}-1} D_{m}+E_{M_{i}}-L E_{s}+k_{M_{i}} E_{s} \leq E_{M_{i}},
$$


where the last inequality is based on (9). Dividing both sides of (10) by $L M_{i}$ and letting $i \rightarrow \infty$ (so as $M_{i}$ ), we have

$$
\lim _{M_{i} \rightarrow \infty} \frac{\sum_{m=1}^{M_{i}} E_{m}}{L M_{i}}-E_{s}+\lim _{M_{i} \rightarrow \infty} \frac{\sum_{m=1}^{M_{i}} k_{m}}{L M_{i}} \leq \lim _{M_{i} \rightarrow \infty} \frac{E_{M_{i}}}{L M_{i}}
$$

Based on the assumption that $\sum_{n=1}^{\infty} P[E>n \epsilon]<\infty$ for any $\epsilon>0$, and Borel-Cantelli lemma [15], we have

$$
\lim _{M_{i} \rightarrow \infty} \frac{E_{M_{i}}}{L M_{i}}=0, \text { a.s. }
$$

Combining (11) with (8) yields

$$
\lim _{N \rightarrow \infty} \frac{K}{N} \leq 0, \text { a.s. }
$$

Since $\frac{K}{N} \geq 0$,(3) is true.

3) Case 3: $P>\frac{E_{s}}{T_{s}}$. Proof by contradiction. Assume $\lim _{i \rightarrow \infty} M_{i}=\infty$. When $E_{c}>E_{s}$, from (8) and (11), we have

$$
\lim _{N \rightarrow \infty} \frac{K}{N} \leq-\left(E_{c}-E_{s}\right)<0, \text { a.s. }
$$

This contradicts with the fact that $\frac{K}{N} \geq 0$; thus, the assumption $\lim _{i \rightarrow \infty} M_{i}=\infty$ cannot be true when $E_{c}>$ $E_{s}$. This means that $M_{i}$ is finite; thus, $K$ is finite as $N \rightarrow$ $\infty$.

The results in Theorem 1 state that there is an asymptotic equivalence between stochastic energy source and deterministic energy source as time goes to infinity, if and only if the average harvested energy in one sample period is no less than the energy required for one sensing operation. This means that the performance of the besteffort sensing policy with stochastic energy sources has the potential to match that of the uniform sensing policy with deterministic energy sources as time becomes large enough and $P \geq \frac{E_{s}}{T_{s}}$. The results hold for a quite general category of energy harvesting processes. It is straightforward to verify that commonly adopted energy harvesting models, such as Poisson energy, Bernoulli energy source, and Gaussian energy source, all fall into this category.

If $P$ is strictly larger than $\frac{E_{s}}{T_{s}}$, then the result is stronger because the number of silent symbols is finite even if the total number of symbols goes to infinity. This means that for almost every energy harvesting sample path, there exists $\bar{N}<\infty$ such that $Q\left(n T_{s}\right) \geq E_{s}, \forall n>\bar{N}$, or there exists a time threshold after which the best-effort sensing policy will be exactly the same as the uniform sensing policy.
The converse portion of Theorem 1 states that $P \geq \frac{E_{s}}{T_{s}}$ is the necessary and sufficient condition for the asymptotic equivalence between the stochastic and deterministic energy sources, because the percentage of silent symbols is non-diminishing if the condition $P \geq \frac{E_{s}}{T_{s}}$ is not satisfied.

Theorem 1 provides the theoretical foundation regarding the asymptotic equivalence between stochastic and deterministic energy sources, with the help of the newly proposed best-effort sensing policy. In the next section, we will show that the asymptotic equivalence can be achieved in a practical sensing system, that is, the proposed best-effort sensing policy with stochastic energy sources can indeed asymptotically achieve the same MSE performance as the uniform sensing policy with constant energy supply.

\section{Asymptotically optimum sensing with the best-effort sensing policy}

This section studies the optimum design and performance analysis of sensing systems employing the newly proposed best-effort sensing policy.

\subsection{Best-effort sensing with stochastic energy sources}

Based on the best-effort sensing policy, define the sensing sample at time instant $k_{n}=n T_{s}$ as

$$
\eta_{n}= \begin{cases}\sqrt{E_{s}} s\left(n T_{s}\right)+z\left(n T_{s}\right), & Q\left(n T_{s}\right) \geq E_{s} \\ z\left(n T_{s}\right), & Q\left(n T_{s}\right)<E_{s}\end{cases}
$$

Define $\eta=\left[\eta_{1}, \cdots, \eta_{N}\right]^{T} \in \mathcal{R}^{N}$, and express (14) in a matrix format as

$$
\eta=\sqrt{E_{s}} \mathbf{x}+\mathbf{z}
$$

where $\mathbf{x}=\left[x_{1}, \cdots, x_{n}\right]^{T}$ with $x_{n}=s\left(n T_{s}\right)$ if $Q\left(n T_{s}\right) \geq E_{s}$ and $x_{n}=0$ otherwise, and $\mathbf{z}=\left[z\left(T_{s}\right), \cdots, z\left(N T_{s}\right)\right]^{T}$. With the best-effort sensing policy, $K$ out of the $N$ elements in $\eta$ contain only noise components.

The system attempts to reconstruct the time-varying random event $s(t)$ by using the observations, $\eta$. Since we are interested in the reconstruction fidelity of a continuous-time random event, the worst case scenario will be considered by estimating $\left\{s\left(n T_{s}+\frac{1}{2} T_{s}\right)\right\}_{n}$, the sequence of points located in the middle between two candidate sensing instants. Define the data vector to be estimated as $\mathbf{d}=\left[s\left(\frac{1}{2} T_{s}\right), s\left(\frac{3}{2} T_{s}\right), \cdots, s\left(N T_{s}-\frac{1}{2} T_{s}\right)\right]^{T}$. It should be noted that $s\left(n T_{s}+\frac{1}{2} T_{s}\right)$ will be estimated even if $x_{n}=0$ and/or $x_{n+1}=0$.

The linear minimum mean squared error (MMSE) estimation of $\mathbf{d}$ based on $\eta$ is

$$
\hat{\mathbf{d}}=\mathbf{W}^{T} \boldsymbol{\eta}
$$


where $\mathbf{W}$ is the MMSE matrix and it satisfies the orthogonal principle

$$
\mathbb{E}\left[\left(\mathbf{W}^{T} \mathbf{y}-\mathbf{d}\right) \mathbf{y}^{T}\right]=\mathbf{0} .
$$

The MMSE matrix is $\mathbf{W}^{T}=\mathbb{E}\left(\mathbf{d} \eta^{T}\right)\left[\mathbb{E}\left(\boldsymbol{\eta} \boldsymbol{\eta}^{T}\right)\right]^{-1}$, which can be expressed as

$$
\mathbf{W}^{T}=\sqrt{E_{s}} \mathbf{R}_{d x}\left[E_{s} \mathbf{R}_{x x}+\mathbf{R}_{z z}\right]^{-1},
$$

where $\mathbf{R}_{d x}=\mathbb{E}\left(\mathbf{d} \mathbf{x}^{T}\right), \mathbf{R}_{x x}=\mathbb{E}\left(\mathbf{x} \mathbf{x}^{T}\right)$, and $\mathbf{R}_{z z}=\sigma_{z}^{2} \mathbf{I}_{N}$ with $\mathbf{I}_{N}$ being a size $N \times N$ identity matrix. If we assume the indices of the sampling instants with $Q\left(n T_{s}\right)<E_{s}$ being $i_{1}, i_{2}, \cdots, i_{K}$, then the $i_{k}$-th column of $\mathbf{R}_{d x}$ is an all-zero column, for $k=1, \cdots, K$. Similarly, the $i_{k}$-th row and the $i_{k}$-th column of $\mathbf{R}_{x x}$ are all-zero vectors, for $k=1, \cdots, K$.

The covariance matrix of the error vector, $\boldsymbol{\epsilon}=\mathbf{d}-\hat{\mathbf{d}}$, can be written as

$$
\mathbf{R}_{\epsilon \epsilon}=\mathbf{R}_{d d}-\mathbf{R}_{d x}\left[\mathbf{R}_{x x}+\frac{\sigma_{z}^{2}}{E_{s}} \mathbf{I}_{N}\right]^{-1} \mathbf{R}_{x d} .
$$

Systems with deterministic energy sources can be considered as a special case of the MMSE receiver described in (15) and (16). With a deterministic energy source, there will be no silent symbols; thus, the system equation can be expressed as

$$
\mathbf{y}=\sqrt{E_{S}} \mathbf{s}+\mathbf{z}
$$

where $\mathbf{y}=\left[y\left(T_{s}\right), \cdots, y\left(N T_{s}\right)\right]^{T}$ and $\mathbf{s}=$ $\left[s\left(T_{s}\right), \cdots, s\left(N T_{s}\right)\right]^{T}$ are the received signal vector and data vector, respectively. Denote the MMSE estimation of $\mathbf{d}$ from $\mathbf{y}$ as $\tilde{\mathbf{d}}=\mathbf{W}_{y}^{T} \mathbf{y}$, where $\mathbf{W}_{y}^{T}$ is the linear MMSE matrix. Similar to (19), the covariance matrix of the error vector, $\mathbf{e}=\mathbf{d}-\tilde{\mathbf{d}}$, can be written as

$$
\mathbf{R}_{e e}=\mathbf{R}_{d d}-\mathbf{R}_{d s}\left[\mathbf{R}_{s s}+\frac{\sigma_{z}^{2}}{E_{s}} \mathbf{I}_{N}\right]^{-1} \mathbf{R}_{s d}
$$

where $\mathbf{R}_{d s}=\mathbb{E}\left(\mathbf{d} \mathbf{s}^{T}\right)$ and $\mathbf{R}_{s s}=\mathbb{E}\left(\mathbf{s s}^{H}\right)$. The crosscovariance matrix $\mathbf{R}_{d s}$ is a Toeplitz matrix with the first row being $\left[r\left(\frac{1}{2} T_{s}\right), r\left(\frac{3}{2} T_{s}\right), \cdots, r\left(N T_{s}-\frac{1}{2} T_{s}\right)\right]$, and the first column $\left[r\left(\frac{1}{2} T_{s}\right), r\left(\frac{1}{2} T_{s}\right), r\left(\frac{3}{2} T_{s}\right), \ldots\right.$, $\left.r\left(N T_{s}-\frac{3}{2} T_{s}\right)\right]^{T}$. The covariance matrix $\mathbf{R}_{s s}$ is a symmetric Toeplitz matrix with the first row being $\left[r(0), r\left(T_{s}\right), \cdots, r\left(N T_{s}-T_{s}\right)\right]$.

The error covariance matrices for systems with stochastic and deterministic energy sources are given in (19) and (21), respectively. The average MSE for systems with stochastic and deterministic energy sources can then be calculated, respectively, as

$$
\begin{aligned}
\sigma_{\epsilon, N}^{2} & =\frac{1}{N} \operatorname{trace}\left(\mathbf{R}_{\epsilon \epsilon}\right), \\
\sigma_{e, N}^{2} & =\frac{1}{N} \operatorname{trace}\left(\mathbf{R}_{e e}\right) .
\end{aligned}
$$

Intuitively, $\sigma_{\epsilon, N}^{2} \geq \sigma_{e, N}^{2}$ since y contains more information than $\eta$. The MSE of the system with uniform sensing and deterministic energy source can serve as a lower bound for systems with stochastic energy sources. However, due to asymptotic equivalence between the stochastic and deterministic energy sources as presented in Theorem 1, we will show in the next subsection that the proposed best-effort sensing policy can asymptotically achieve the same performance as the uniform sensing policy as $N \rightarrow \infty$.

\subsection{Asymptotic achievability of the MSE lower bound}

The asymptotic equivalence between the two sensing schemes relies on the asymptotic equivalence between sequences of matrices, which is defined as follows.

Definition 2 (Asymptotical equivalence [16]). Two sequences of $N \times N$ matrices, $\left\{\mathbf{A}_{N}\right\}_{N}$ and $\left\{\mathbf{B}_{N}\right\}_{N}$ are asymptotically equivalent if

1. $\mathbf{A}_{N}$ and $\mathbf{B}_{N}$ are bounded in strong norm:

$$
\left\|\mathbf{A}_{N}\right\|,\left\|\mathbf{B}_{N}\right\|<C<\infty, N=1,2, \cdots
$$

where $\|\mathbf{A}\|=\max _{k} \sqrt{\lambda_{k}}$ is the strong norm of $\mathbf{A}, \lambda_{k}$ is the eigenvalue of $\mathbf{A}^{H} \mathbf{A}$, and $\mathbf{A}^{H}$ is the Hermitian of the matrix $\mathbf{A}$.

2. $\mathbf{A}_{N}-\mathbf{B}_{N}$ goes to zero in weak norm as $N \rightarrow \infty$

$$
\lim _{N \rightarrow \infty}\left|\mathbf{A}_{N}-\mathbf{B}_{N}\right|=0,
$$

where $\left|\mathbf{A}_{N}\right|=\sqrt{\frac{1}{N} \sum_{m=1}^{N} \sum_{n=1}^{N}\left|a_{m n}\right|^{2}}$ is the weak norm of $\mathbf{A}_{N}$ with $a_{m n}$ being the $(m, n)$-th element of the matrix $\mathbf{A}_{N}$.

The asymptotic equivalence between two sequences of matrices, $\left\{\mathbf{A}_{N}\right\}$ and $\left\{\mathbf{B}_{N}\right\}$, is denoted as $\mathbf{A}_{N} \sim \mathbf{B}_{N}$.

Lemma 1. Consider two sequences of matrices, $\left\{\boldsymbol{A}_{N}\right\}_{N}$ and $\left\{\boldsymbol{B}_{N}\right\}_{N}$. The squares of the elements on any row or column of the two matrices are absolutely summable as $N \rightarrow \infty$. If the two matrices differ in $K_{1}$ rows and $K_{2}$ columns, and $\lim _{N \rightarrow \infty} \frac{K_{1}+K_{2}}{N}=0$, then $\boldsymbol{A}_{N} \sim \boldsymbol{B}_{N}$.

Proof. Denote the $(m, n)$-th elements of $\mathbf{A}_{N}$ and $\mathbf{B}_{N}$ as $a_{m n}$ and $b_{m n}$, respectively. Since the squares of the elements on each row or column of the two matrices are absolutely summable, the matrices are bounded in strong norm. Assume the two matrices differ in rows $r_{1}, \cdots, r_{K_{1}}$ and columns $c_{1}, \cdots, c_{K_{2}}$, then

$$
\left|\mathbf{A}_{N}-\mathbf{B}_{N}\right|^{2} \leq \frac{1}{N}\left[\sum_{k=1}^{K_{1}} \sum_{n=1}^{N}\left|a_{r_{k} n}-b_{r_{k}}\right|^{2}+\sum_{m=1}^{M} \sum_{k=1}^{K_{2}}\left|a_{m c_{k}}-b_{m c_{k}}\right|^{2}\right]
$$


Since the squares of the elements on any row or column are absolutely summable, then there exists $C>0$ such that

$$
\begin{gathered}
\sum_{n=1}^{\infty}\left|a_{r_{k} n}-b_{r_{k} n}\right|^{2} \leq \sum_{n=1}^{\infty}\left|a_{r_{k} n}\right|^{2}+\sum_{n=1}^{\infty}\left|b_{r_{k} n}\right|^{2}<2 C \\
\sum_{m=1}^{\infty}\left|a_{m c_{k}}-b_{m c_{k}}\right|^{2} \leq \sum_{m=1}^{\infty}\left|a_{m c_{k}}\right|^{2}+\sum_{m=1}^{\infty}\left|b_{m c_{k}}\right|^{2}<2 C
\end{gathered}
$$

Thus,

$$
\lim _{N \rightarrow \infty}\left|\mathbf{A}_{N}-\mathbf{B}_{N}\right|<\sqrt{4 C} \lim _{N \rightarrow \infty} \sqrt{\frac{K}{N}}
$$

Since $\lim _{N \rightarrow \infty} \frac{K}{N}=0$, it is straightforward that $\lim _{N \rightarrow \infty}\left|\mathbf{A}_{N}-\mathbf{B}_{N}\right|=0$.

From (19) and (21), $\sigma_{\epsilon}^{2}$ and $\mathbf{R}_{\epsilon \epsilon}$ depend on $\mathbf{R}_{d x}$ and $\mathbf{R}_{x x}$, while $\sigma_{e}^{2}$ and $\mathbf{R}_{e e}$ depend on $\mathbf{R}_{d s}$ and $\mathbf{R}_{s s}$. We have the following lemma regarding the relationships between the two groups of matrices as $N \rightarrow \infty$.

Lemma 2. If $P \geq \frac{E_{s}}{T_{s}}$ in the best-effort sensing policy, then $\boldsymbol{R}_{d x} \sim \boldsymbol{R}_{d s}$, and $\boldsymbol{R}_{x x} \sim \boldsymbol{R}_{s s}$.

Proof. All matrices are absolutely summable in each row or column. Based on the best-effort sensing policy, $\mathbf{x}$ can be obtained by replacing $K$ elements in $\mathbf{s}$ with zeros. Since $P \geq \frac{E_{s}}{T_{s}}$, we have $\lim _{N \rightarrow \infty} \frac{K}{N}=0$ a.s.

If we assume the indices of the sampling instants with $Q\left(n T_{s}\right)<E_{s}$ being $i_{1}, i_{2}, \cdots, i_{K}$, then $\mathbf{R}_{d x}$ can be obtained by replacing the $i_{k}$-th column of $\mathbf{R}_{d s}$ with an all-zero column, for $k=1, \cdots, K$. From Lemma 1, $\mathbf{R}_{d x} \sim \mathbf{R}_{d s}$.

Similarly, $\mathbf{R}_{x x}$ can be obtained by replacing the $i_{k}$-th row and the $i_{k}$-th column of $\mathbf{R}_{s s}$ with all-zero vectors, for $k=$ $1, \cdots, N$. From Lemma $1, \mathbf{R}_{x x} \sim \mathbf{R}_{s s}$.

Now we are ready to present the second main result of this paper in the following theorem, which states the asymptotic equivalence between systems with stochastic and deterministic energy sources in terms of MSE performance.

Theorem 2. Consider two sensing systems, one with the best-effort sensing policy and a stochastic energy source as described in (15), and one with the uniform sensing policy and a deterministic energy source as described in (20). If $P \geq \frac{E_{s}}{T_{s}}$, then

$$
\lim _{N \rightarrow \infty} \sigma_{\epsilon, N}^{2}=\lim _{N \rightarrow \infty} \sigma_{e, N}^{2}=\sigma_{0}^{2}
$$

where $\sigma_{\epsilon, N}$ and $\sigma_{e, N}^{2}$ are the MSE of the two systems defined in (22a) and (22b), respectively. Specifically, with the power-law time covariance in (1)

$$
\sigma_{0}^{2}=\left[\frac{\sigma_{z}^{2}}{E_{s}}+\frac{1-\rho^{T_{s}}}{1+\rho^{T_{s}}}\right]^{\frac{1}{2}}\left[\frac{\sigma_{z}^{2}}{E_{s}}+\frac{1+\rho^{T_{s}}}{1-\rho^{T_{s}}}\right]^{-\frac{1}{2}}
$$

Proof. The Toeplitz matrix, $\mathbf{R}_{d s}$, is uniquely determined by the sequence $\mathbf{r}_{d s}=\left[r_{-(N-1)}, \cdots, r_{0}, \cdots, r_{N-1}\right]^{T}$, where $r_{n}=\rho^{\frac{T_{s}}{2}} \rho^{-(n+1) T_{s}}$ when $n<0$, and $r_{n}=\rho^{\frac{T_{s}}{2}} \rho^{n T_{s}}$ otherwise. When $N \rightarrow \infty$, the discrete time Fourier transform (DTFT) of the sequence $\mathbf{r}_{d s}$ can be calculated as

$$
\Lambda_{d s}(f)=\rho^{\frac{T_{s}}{2}} \frac{\left(1-\rho^{T_{s}}\right)\left(1+e^{j 2 \pi f}\right)}{1+\rho^{2 T_{s}}-2 \rho^{T_{s}} \cos (2 \pi f)} .
$$

Based on [17] (Lemma 2), $\mathbf{R}_{d s}$ is asymptotically equivalent to a circulant matrix, $\mathbf{C}_{d s}=\mathbf{U}_{N}^{H} \mathbf{D}_{d s} \mathbf{U}_{N}$, where $\mathbf{U}_{N}$ is the unitary discrete Fourier transform (DFT) matrix with the $(m, n)$-th element being $\left(\mathbf{U}_{N}\right)_{m, n}=$ $\frac{1}{\sqrt{N}} \exp \left[-j 2 \pi \frac{(m-1)(n-1)}{N}\right]$, and $\mathbf{D}_{d s}$ is a diagonal matrix with its $k$-th diagonal element being $\left(\mathbf{D}_{d s}\right)_{k, k}=$ $\Lambda_{d s}\left(\frac{k-1}{N}\right)$.

Similarly, the symmetric Toeplitz matrix, $\mathbf{R}_{s S}$, is uniquely determined by the sequence $\boldsymbol{\phi}_{s s}=$ $\left[\phi_{-(N-1)}, \cdots, \phi_{0}, \cdots, \phi_{N-1}\right]^{T}$, where $\phi_{n}=\rho^{|n| T_{s}}$. When $N \rightarrow \infty$, the DTFT of the sequence $\phi_{s s}$ can be calculated as

$$
\Lambda_{s s}(f)=\frac{1-\rho^{2 T_{s}}}{1+\rho^{2 T_{s}}-2 \rho_{s}^{T} \cos (2 \pi f)} .
$$

The symmetric Toeplitz matrix $\mathbf{R}_{s s}$ is asymptotically equivalent to a circulant matrix, $\mathbf{C}_{s s}=\mathbf{U}_{N}^{H} \mathbf{D}_{s s} \mathbf{U}_{N}$, where $\mathbf{D}_{s s}$ is a diagonal matrix with its $k$-th diagonal element being $\left(\mathbf{D}_{s s}\right)=\Lambda_{s s}\left(\frac{k-1}{N}\right)$.

When $P \geq \frac{E_{s}}{T_{s}}$, we have $\lim _{N \rightarrow \infty} \frac{K}{N}=0$ from Theorem 1. Based on Lemma 2 and [16] (Theorem 2.1), we have

$$
\begin{aligned}
\mathbf{R}_{d x} & \sim \mathbf{R}_{d s} \sim \mathbf{C}_{s s} \\
\mathbf{R}_{x x} & \sim \mathbf{R}_{s s} \sim \mathbf{C}_{s s}
\end{aligned}
$$

Therefore, based on [16] (Theorem 2.1), we have $\mathbf{R}_{\epsilon \epsilon} \sim$ $\mathbf{R}_{e e} \sim \mathbf{C}_{e e}$, where $\mathbf{C}_{e e}$ is a circulant matrix defined as

$$
\mathbf{C}_{e e}=\mathbf{C}_{s s}-\mathbf{C}_{d s}\left[\mathbf{C}_{s s}+\frac{\sigma_{z}^{2}}{E_{s}} \mathbf{I}_{N}\right]^{-1} \mathbf{C}_{d s}^{H}
$$

The circulant matrix $\mathbf{C}_{e e}$ can be expressed as $\mathbf{C}_{e e}=$ $\mathbf{U}_{N}^{H} \mathbf{D}_{e e} \mathbf{U}_{N}$, where $\mathbf{D}_{e e}$ is a diagonal matrix defined as

$$
\mathbf{D}_{e e}=\mathbf{D}_{s s}-\mathbf{D}_{d s}\left[\mathbf{D}_{s s}+\frac{\sigma_{z}^{2}}{E_{s}} \mathbf{I}_{N}\right]^{-1} \mathbf{D}_{d s}^{H} .
$$

Therefore, both error covariance matrices are asymptotically equivalent to the same circulant matrix. Based 
on Szego's theorem [17], when $N \rightarrow \infty$, we have

$$
\lim _{N \rightarrow \infty} \sigma_{\epsilon, N}^{2}=\lim _{N \rightarrow \infty} \sigma_{e, N}^{2}=\int_{-\frac{1}{2}}^{\frac{1}{2}}\left[\Lambda_{s s}(f)-\frac{\left|\Lambda_{d s}(f)\right|^{2}}{\Lambda_{s s}(f)+\frac{\sigma_{z}^{2}}{E_{s}}}\right] d f
$$

Substituting (30) and (29) into the above equation and simplifying yields (28).

Theorem 2 states that systems with the proposed besteffort sensing policy and stochastic energy sources can asymptotically achieve the same MSE performance as systems with the uniform sensing policy and deterministic energy sources, as long as the energy consumption for each sensing operation is not more than the average harvested energy in each sampling period. Theorem 1 provides the theoretical foundation on the asymptotic equivalence between stochastic and deterministic energy sources, and Theorem 2 demonstrates that such equivalence can be achieved in practical systems.

\section{Numerical and simulation results}

Numerical and simulation results are presented in this section to demonstrate the asymptotic equivalence between systems with stochastic and deterministic energy sources.

Figure 1 shows the value of $\frac{K}{N}$ as a function of $N$ for systems with various stochastic energy sources, where $K$ is the number of silent sensing symbols and $N$ is the total number of candidate sensing symbols. All systems employ the best-effort sensing policy proposed in this paper. The convergence behaviors of $\frac{K}{N}$ are demonstrated for $P=$ $0.9 \frac{E_{s}}{T_{s}}, P=\frac{E_{s}}{T_{s}}$, and $P=1.1 \frac{E_{s}}{T_{s}}$, respectively. The simulation

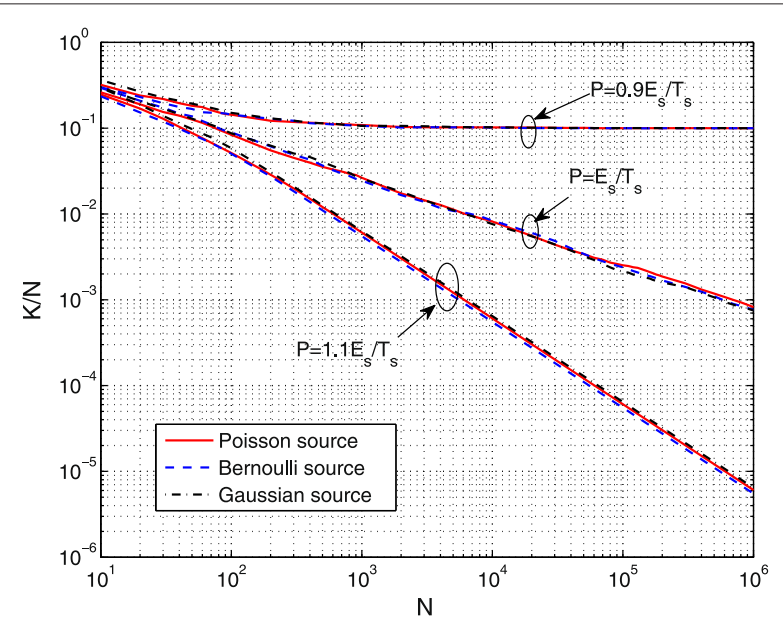

Figure $1 K / N$ for various energy sources. results are obtained by averaging over 100 independent runs for each configuration. All three energy sources have similar convergence behaviors as $N$ increases. When $P \geq$ $\frac{E_{S}}{T_{s}}$ and $N>100$, the value of $\log \frac{K}{N}$ decreases almost linearly with respect to $\log N$, and the absolute slope increases as $\frac{P T_{s}}{E_{s}}$ increases. The simulation results conform to Theorem 1 , which states that $\lim _{N \rightarrow \infty} \frac{K}{N}=0$ when $P \geq \frac{E_{s}}{T_{s}}$. On the other hand, when $P=0.9 \frac{E_{s}}{T_{s}}, \frac{K}{N}$ tends to a constant value 0.1 , as $N \rightarrow \infty$. The constant value is the same as the lower bound, $1-\frac{P T_{s}}{E_{s}}$, predicted by Theorem 1. The results in Figure 1 demonstrate through simulations that the percentage of silent sensing symbols diminishes as time goes to infinity, as long as the average energy harvesting rate is no less than the average energy consumption rate. The actual distributions of the energy sources have no impact on the convergence behavior.

Figure 2 compares the MSE performance of systems with both stochastic and deterministic energy sources. The best-effort sensing policy is employed by systems with stochastic energy sources, and uniform sensing is employed by systems with deterministic energy sources. The stochastic energy sources are the Gaussian sources with mean $P=\frac{E_{s}}{T_{s}}$. It is assumed that all systems have the same average power $P=\frac{E_{s}}{T_{s}}$ with the normalized signalto-noise ratio (SNR) $\gamma_{0}=\frac{P}{\sigma_{z}^{2}}$ being $0 \mathrm{~dB}$. The power-law coefficient is $\rho=0.9$. The simulation results are obtained through Monte Carlo simulations, where the data are generated as a zero-mean Gaussian random process with the covariance function satisfying the power-law relationship. In the simulations, the data are sampled and then estimated, and the estimation results are compared to the true values to calculate the MSE. Each point on the simulation MSE curves is obtained by averaging over 2,000 independent runs for each configuration. It can be seen that

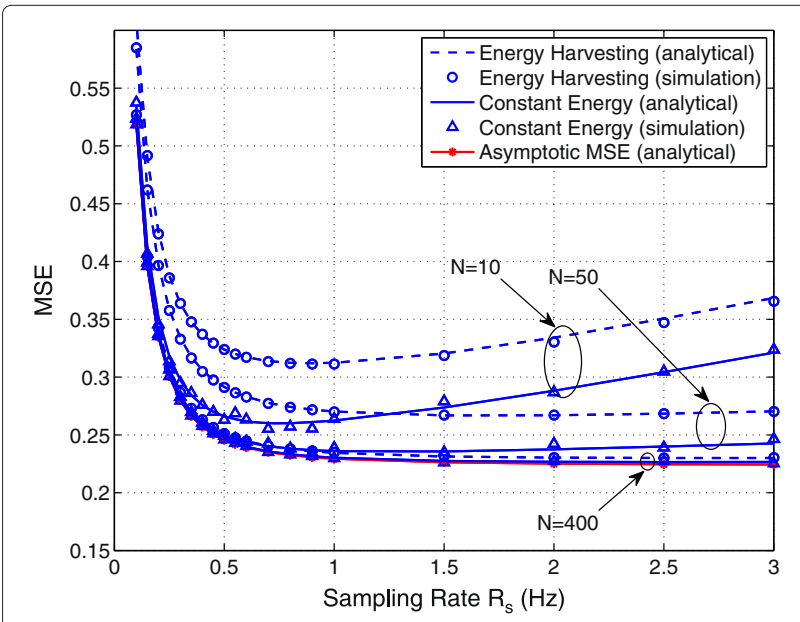

Figure 2 MSE as a function of the sampling frequency $(\rho=0.9)$. 
the simulation results match perfectly with their analytical counterparts. As expected, the MSE performance of systems with deterministic energy sources is consistently better than that of systems with stochastic energy sources. However, the performance gap narrows as $N$ increases. When $N=400$, the MSE performance of the two systems are almost identical, and they coincide with the asymptotic MSE obtained with $N \rightarrow \infty$. Therefore, when $N$ is sufficiently large, the best-effort sensing policy can achieve a performance that is almost the same as the uniform sensing policy. Thus, the results in Figure 2 demonstrate the asymptotic equivalence between stochastic and deterministic energy sources in a practical system.

In addition, it can be seen from Figure 2 that the MSE is convex when $N$ is small $(N=10$ or 50$)$, and it becomes a monotonically decreasing function when $N$ is large $(N=$ 400). Under a fixed power, a larger sampling rate means less energy per sample, which might degrade the system performance. On the other hand, a larger sampling rate means a stronger correlation between two adjacent samples, which contributes positively to the MSE performance. Therefore, changing the sampling rate results in different trade-offs between energy per sample and sample correlations. When $N$ is large enough, increasing the sampling rate beyond a certain threshold (e.g., $R_{s}=1$ $\mathrm{Hz}$ when $N=400$ ) has negligible impact on the MSE performance.

Figure 3 shows the simulation and analytical MSE of systems with stochastic energy sources as functions of the SNR. The sampling rate is $1 \mathrm{~Hz}$. The analytical results with finite $N$ are calculated from (22). The simulation results are obtained through Monte Carlo simulations. Each point on the curve is averaged over 2,000 independent simulation runs. The simulation results match the analytical results very well. As expected, the MSE

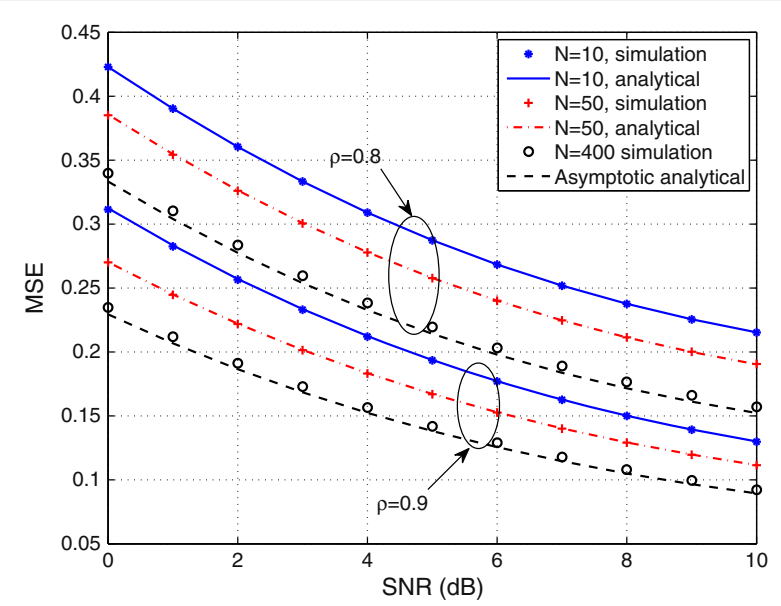

Figure 3 MSE as a function of the SNR for systems with stochastic energy sources $\left(R_{s}=1 \mathrm{~Hz}\right)$. decreases as $\rho$ or $N$ increases. In addition, the MSE with $N=400$ is almost the same as the asymptotic MSE obtained with $N \rightarrow \infty$.

\section{Conclusions}

The asymptotic equivalence between stochastic and deterministic energy sources has been demonstrated through both theoretical analysis and practical examples. To account for the stochastic nature of energy harvested from the ambient environment, a best-effort sensing policy has been proposed for energy harvesting sensing systems. It has been shown that the difference between the best-effort sensing scheme and the ideal uniform sensing scheme diminishes as time goes to infinity, if and only if the average energy collection rate is no less than the average energy consumption rate, regardless of the actual distribution of the stochastic energy source. The asymptotic equivalence has been used for the development of an optimum energy harvesting sensing system. It has been shown through both theoretical analysis and simulation results that systems with the best-effort sensing scheme and stochastic energy sources can achieve almost the same MSE performance as systems with uniform sensing and deterministic energy sources when the number of samples is greater than 400 .

\section{Competing interests}

The authors declare that they have no competing interests.

\section{Acknowledgements}

This work was supported in part by the U.S. National Science Foundation under Grants ECCS-1202075, ECCS-1405403, and ECCS-1454471.

Received: 20 September 2014 Accepted: 10 March 2015

Published online: 26 March 2015

\section{References}

1. J Yang, S Ulukus, Optimal packet scheduling in an energy harvesting communication system. IEEE Trans. Comm. 60(1), 220-230 (2012)

2. MA Antepli, E Uysal-Biyikoglu, H Erkal, Optimal packet scheduling on an energy harvesting broadcast link. IEEE J. Sel. Areas Commun. 29(8), 1721-1731 (2011)

3. J Yang, O Ozel, S Ulukus, Broadcasting with an energy harvesting rechargeable transmitter. IEEE Trans. Wireless Comm. 11(2), 571-583 (2012)

4. J Yang, S Ulukus, Optimal packet scheduling in a multiple access channel with energy harvesting transmitters. J. Commun. Networks. 14, 140-150 (2012)

5. O Ozel, KTutuncuoglu, J Yang, S Ulukus, A Yener, Transmission with energy harvesting nodes in fading wireless channels: optimal policies. IEEE J Sel. Areas Commun. 29(8), 1732-1743 (2011)

6. KTutuncuoglu, A Yener, Sum-rate optimal power policies for energy harvesting transmitters in an interference channel. J. Commun. Networks. 14(2), 151-161 (2012)

7. G Yang, V Tan, CK Ho, SH Ting, YL Guan, Wireless compressive sensing for energy harvesting sensor nodes. Signal Process., IEEE Trans. 61 (18), 4491-4505 (2013)

8. J Geng, L Lai, Non-Bayesian quickest change detection with stochastic sample right constraints. IEEE Trans. Signal Process. 61(20) (2013)

9. N Sun, J Wu, Optimum sampling in spatial-temporally correlated wireless sensor networks. EURASIP J. Wireless Commun. Networking. 2013, 5

10. J Wu, N Sun, Optimum sensor density in distortion-tolerant wireless sensor networks. Wireless Commun., IEEE Trans. 11(6), 2056-2064 (2012) 
11. J Wu, J Yang, in Proc. IEEE Global Telecommun. Conf. Globecom'14. The asymptotic equivalence between sensing systems with energy harvesting and conventional energy sources (Austin, TX, USA, 8 December 2014)

12. J Yang, J Wu, in Proc. IEEE Global Telecommun. Conf. Globecom'14. Optimal sampling of random processes under stochastic energy constraints (Austin, TX, USA, 8 December 2014)

13. J Yang, $X$ Wu, J Wu, in Proc. IEEE Intern. Conf. Commun. (ICC'15). Adaptive sensing scheduling for energy harvesting sensors with finite battery (London, UK, 8 June 2015)

14. K Ritter, GW Wasilkowski, H Wozniakowski, Multivariate integration and approximation of random fields satisfying Sacks-Ylvisaker conditions. Ann. Appl. Probability. 5, 518-540 (1995)

15. A Klenke, Probability Theory: A Comprehensive Course (Universitext). (Springer, New York, 2007)

16. RM Gray, Toeplitz and circulant matrices: a review. Foundations and Trends in Commun. and Inf. Theory. 2(3), 155-239 (2006)

17. H Gazzah, P Regalia, J-P Delmas, Asymptotic eigenvalue distribution of block Toeplitz matrices and application to blind SIMO channe identification. Inf. Theory, IEEE Trans. 47(3), 1243-1251 (2001)

\section{Submit your manuscript to a SpringerOpen ${ }^{\mathcal{O}}$ journal and benefit from:}

- Convenient online submission

- Rigorous peer review

- Immediate publication on acceptance

- Open access: articles freely available online

- High visibility within the field

- Retaining the copyright to your article

Submit your next manuscript at $\gg$ springeropen.com 